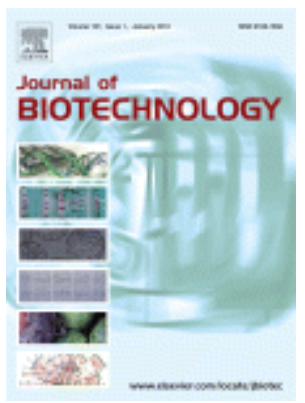

\title{
A new cloning system based on the Oprl lipoprotein for the production of recombinant bacterial cell wall-derived immunogenic formulations
}

- Afonso P. Basto ${ }^{a}$,

- João Piedade

- Ruben Ramalho ${ }^{c, d}$,

- Susana Alves ${ }^{\mathrm{e}, \mathrm{f}}$,

- Helena Soares ${ }^{d, g}, \underline{h}$,

- Pierre Cornelis ${ }^{\text {, }}$

- Carlos Martins ${ }^{a}$,

- Alexandre Leitão

- $\quad$ a Laboratório de Doenças Infecciosas, CIISA, Faculdade de Medicina Veterinária, Universidade Técnica de Lisboa, Avenida da Universidade Técnica, 1300-477 Lisboa, Portugal

- ${ }^{\mathrm{b}}$ Grupo de Virologia/UPMM, UEI de Microbiologia Médica, Instituto de Higiene e Medicina Tropical, Universidade Nova de Lisboa, Rua da Junqueira 100, 1349008 Lisboa, Portugal

- ' Instituto Superior Técnico, 1049-001 Lisboa, Portugal

- ${ }^{d}$ Instituto Gulbenkian de Ciência, 2781-901 Oeiras, Portugal

- $\quad$ e Instituto Nacional dos Recursos Biológicos, INRB, UPA-INIA, Fonte-Boa, 2005048 Vale de Santarém, Portugal

- $\quad{ }^{\mathrm{f}}$ REQUIMTE, ICBAS, Instituto de Ciências Biomédicas de Abel Salazar, Universidade do Porto, 4485-661 Vairão, Portugal 
- ${ }^{g}$ Centro de Química e Bioquímica, Faculdade de Ciências, Universidade de Lisboa, 1749-016 Lisboa, Portugal

- $\quad$ h Escola Superior de Tecnologia da Saúde de Lisboa, 1990-096 Lisboa, Portugal

- 'Department of Bioengineering Sciences, Research group Microbiology, and VIB Department of Structural Biology, Vrije Universiteit Brussel, Pleinlaan 2, 1050 Brussels, Belgium

- $\quad{ }^{j}$ Instituto de Investigação Científica Tropical, CVZ, CIISA, Avenida da Universidade Técnica, 1300-477 Lisboa, Portugal

- http://dx.doi.org/10.1016/j.jbiotec.2011.11.006, How to Cite or Link Using DOI

- $\quad$ Permissions \& Reprints

\section{Abstract}

The conjugation of antigens with ligands of pattern recognition receptors (PRR) is emerging as a promising strategy for the modulation of specific immunity. Here, we describe a new Escherichia coli system for the cloning and expression of heterologous antigens in fusion with the Oprl lipoprotein, a TLR ligand from the Pseudomonas aeruginosa outer membrane (OM). Analysis of the Oprl expressed by this system reveals a triacylated lipid moiety mainly composed by palmitic acid residues. By offering a tight regulation of expression and allowing for antigen purification by metal affinity chromatography, the new system circumvents the major drawbacks of former versions. In addition, the anchoring of Oprl to the OM of the host cell is further explored for the production of novel recombinant bacterial cell wall-derived formulations (OM fragments and OM vesicles) with distinct potential for PRR activation. As an example, the African swine fever virus ORF A104R was cloned and the recombinant antigen was obtained in the three formulations. Overall, our results validate a new system suitable for the production of immunogenic formulations that can be used for the development of experimental vaccines and for studies on the modulation of acquired immunity.

\section{Highlights}

We propose a new system for expression of antigens fused with the Oprl lipoprotein. It offers tight control of expression and metal affinity chromatography purification. The Oprl expressed by the system is triacylated, mainly by palmitic acid residues. The cloned antigens can be obtained in three bacterial cell wall-derived formulations. Applications include studies on immunization and on modulation of acquired immunity.

\section{Abbreviations}


- ALFA, amide-linked fatty acid;

- ASFV, African swine fever virus;

- $\quad \mathrm{CTL}$, cytotoxic T lymphocyte;

- DDM, n-dodecyl- $\beta$-d-maltopyranoside;

- $D M, n$-decyl- $\beta$-d-maltopyranoside;

- ELFA, ester-linked fatty acid;

- FA, fatty acid;

- GLC, gas-liquid chromatography;

- IPTG, isopropyl- $\beta$-d-thiogalactopyranoside;

- MCS, multiple cloning site;

- MHC, major histocompatibility complex;

- MS, mass spectrometry;

- OG, octyl $\beta$-d-glucopyranoside;

- OM, outer membrane;

- OMF, outer membrane fragments;

- OMV, outer membrane vesicles;

- Oprl, outer membrane lipoprotein I;

- PMSF, phenylmethylsulfonyl fluoride;

- PRR, pattern recognition receptors;

- TCA, trichloroacetic acid;

- TLR, Toll-like receptors

\section{Keywords}

- Outer membrane lipoprotein I;

- Pseudomonas;

- Bacterial outer membrane proteins;

- Cloning vector;

- Immunomodulation;

- Adjuvant 